\title{
Study on the Neutrino Oscillation with a Next Generation Medium-Baseline Reactor Experiment
}

\author{
Chang Dong Shin and Kyung Kwang Joo \\ Department of Physics, Chonnam National University, Gwangju 500-757, Republic of Korea \\ Correspondence should be addressed to Kyung Kwang Joo; kkjoo@chonnam.ac.kr
}

Received 25 February 2014; Accepted 15 April 2014; Published 12 May 2014

Academic Editor: Theocharis Kosmas

Copyright (c) 2014 C. D. Shin and K. K. Joo. This is an open access article distributed under the Creative Commons Attribution License, which permits unrestricted use, distribution, and reproduction in any medium, provided the original work is properly cited. The publication of this article was funded by $\mathrm{SCOAP}^{3}$.

\begin{abstract}
For over fifty years, reactor experiments have played an important role in neutrino physics, in both discoveries and precision measurements. One of the methods to verify the existence of neutrino is the observation of neutrino oscillation phenomena. Electron antineutrinos emitted from a reactor provide the measurement of the small mixing angle $\theta_{13}$, providing rich programs of neutrino properties, detector development, nuclear monitoring, and application. Using reactor neutrinos, future reactor neutrino experiments, more precise measurements of $\theta_{12}, \Delta m_{12}^{2}$, and mass hierarchy will be explored. The precise measurement of $\theta_{13}$ would be crucial for measuring the $\mathrm{CP}$ violation parameters at accelerators. Therefore, reactor neutrino physics will assist in the complete understanding of the fundamental nature and implications of neutrino masses and mixing. In this paper, we investigated several characteristics of RENO-50, which is a future medium-baseline reactor neutrino oscillation experiment, by using the GloBES simulation package.
\end{abstract}

\section{Introduction}

Over the last decade, great progress has been made in understanding the neutrino sector of elementary particle physics. The discovery of neutrino oscillations is a direct indication of physics beyond the standard model. It provides a unique new window to explore physics at the Grand Unification Energy scale. While the absolute neutrino mass has not yet been measured, neutrino oscillation implies that neutrinos have a nonzero mass and are mixed together. Neutrino oscillations are described by the three PontecorvoMaki-Nakagawa-Sakata (PMNS) mixing angles $\left(\theta_{12}, \theta_{23}\right.$, and $\left.\theta_{13}\right)$, one $\mathrm{CP}$ violating phase, and two independent mass squared differences $\left(\Delta m_{31}^{2}, \Delta m_{21}^{2}\right)[1,2]$. The mixing angles, $\left(\theta_{12}, \theta_{23}\right.$, and $\left.\theta_{13}\right)$, have been measured using atmospheric, solar, accelerator beam, and reactor neutrino experiments. Among the mixing angles, $\left(\theta_{12}, \theta_{23}\right)$, the values are relatively well measured. $\theta_{12}$ was measured using solar neutrinos and the KamLAND experiment $[3,4]$, while $\theta_{23}$ was measured using atmospheric neutrinos and the long baseline accelerator $\mathrm{K} 2 \mathrm{~K}$ experiment $[5,6]$. However, for the $\theta_{13}$ value, until the year 2012, the best upper limit was set by the
Chooz reactor antineutrino disappearance experiment [7]. The reactor experiments (Double Chooz, Daya Bay, and RENO) have measured $\theta_{13}$ and provide accurate information on $\sin ^{2} 2 \theta_{13}$ [8-10]. In addition, reactor neutrino experiments make accurate measurements of reactor neutrino fluxes and spectra to search for sterile neutrinos [11-13]. Also, future accelerator based neutrino experiments will provide a rich program of measuring $\mathrm{CP}$ violation and matter effects. However, in the long baseline experiments, degeneracies and parameter correlations occur among $\theta_{13}$, the CP violation phase $\left(\delta_{\mathrm{CP}}\right)$, neutrino mass hierarchy, and $\theta_{23}$. The possibility of measuring the $\mathrm{CP}$ violation effect can be fulfilled only if the value of $\theta_{13}$ is precisely measured. Combining the results from the accelerator and reactor-based experiments could offer the first glimpse of $\delta_{\mathrm{CP}}$ without the necessity for long running accelerators with antineutrino beams [14].

After the RENO experiment, RENO collaboration plans to construct an underground detector of RENO-50 consisting of 18,000 tons of ultralow-radioactivity unloaded liquid scintillator (LS) and high quantum efficiency (QE) photomultiplier tubes (PMTs). At $\sim 50 \mathrm{~km}$ from the reactor center, the neutrino oscillation takes place maximally due to $\theta_{12}$. An 


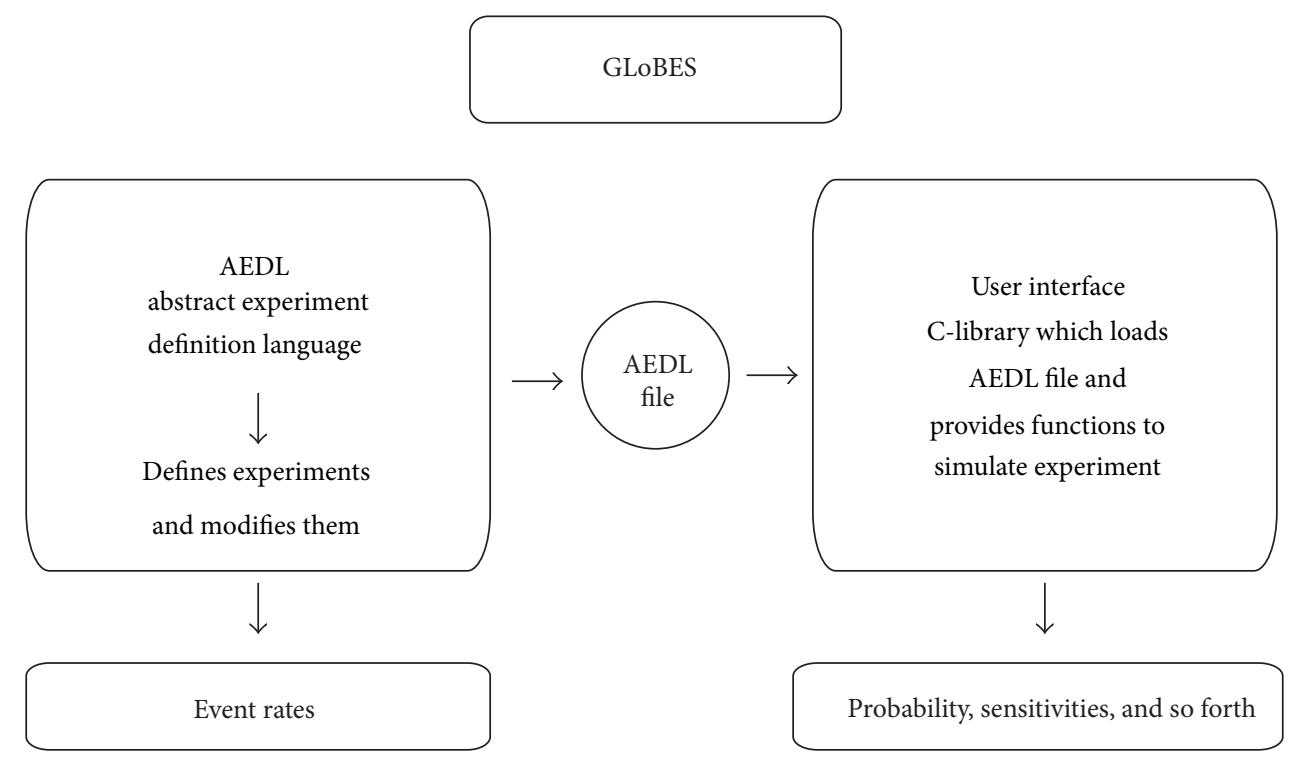

FIGURE 1: Schematics of GLoBES package, which mainly consists of two parts: AEDL and user interface C-library.

experiment with the baseline of $\sim 50 \mathrm{~km}$ could be a natural extension of the current RENO $\theta_{13}$ experiment.

For this study, we used the general long baseline experiment simulator (GLoBES) [15]. Developed by Patrick Huber, Joachim Kopp, Manfred Lindner, Mark Rolinec, and Walter Winter, GLoBES is a computer-based simulator used for long baseline neutrino oscillation by setting a neutrino source, baseline, and detector. GLoBES is the only open source software and is based on the C-library language. As shown in Figure 1, GLoBES consists of two parts. Firstly, abstract experiment definition language (AEDL) provides the experimental setup for neutrino sources. Secondly, the Clibrary corresponds to the detector for processing to provide oscillation probabilities, rate vectors, and $\chi^{2}$-values.

\section{Results from RENO}

2.1. Experimental Setup. The Reactor Experiment for Neutrino Oscillation (RENO) is a reactor-based neutrino oscillation experiment to measure the smallest neutrino mixing angle $\left(\theta_{13}\right)$ using electron antineutrinos emitted from the Hanbit (previously named Yonggwang) nuclear power plant in Korea. It is located on the west coast of the southern part of Korea, about $300 \mathrm{~km}$ from Seoul. The power plant consists of six pressurized water reactors producing a total thermal power of $16.4 \mathrm{GW}_{\text {th }}$. The six reactors, each with a maximum thermal output of $2.8 \mathrm{GW}_{\text {th }}$ (reactors $3,4,5$, and 6) or $2.9 \mathrm{GW}_{\text {th }}$ (reactors 1 and 2), are aligned roughly equal distances and span $\sim 1.3 \mathrm{~km}$. The RENO uses two identical near and far detectors to reduce systematic uncertainties. The near and far detectors are placed approximately $290 \mathrm{~m}$ and $1.4 \mathrm{~km}$ away, respectively, from the center of the reactor array. The near detector (ND) is located under a $70 \mathrm{~m}$ high hill with an overburden of $\sim 110$ m.w.e. (meterwater equivalent) and the far detector (FD) is placed under a $260 \mathrm{~m}$ high mountain with an overburden of $\sim 450$ m.w.e. $[10,16]$.
2.2. Result on $\theta_{13}$ Measurement. Data-taking began at RENO in August, 2011. A clear disappearance of reactor antineutrinos is observed. At RENO, the value of $\sin ^{2} 2 \theta_{13}$ with two identical detectors has been successfully measured. The measured value using rate-only analysis is $\sin ^{2} 2 \theta_{13}=$ $0.100 \pm 0.010$ (stat.) \pm 0.015 (sys.), corresponding to $6.3 \sigma$ significance for 403 days data [10]. Therefore, the current size of the total error is \pm 0.018 [10]. Based on the next three years of projected data-taking, the statistical error will be \pm 0.006 and the systematic error will reach \pm 0.005 . Therefore, RENO will reach $\sin ^{2} 2 \theta_{13}$ at an $8 \%$ precision level.

In current RENO environment, there are mainly three types of backgrounds which mimic IBD signals: accidental, fast neutron and the ${ }^{9} \mathrm{Li} /{ }^{8} \mathrm{He}$ backgrounds. The accidental backgrounds are caused by external gammas such as radioactivity from detector and environment. The estimated accidental background rate is $3.61 \pm 0.05(0.60 \pm 0.03)$ events/day for the ND (FD) [10]. In addition, when atmospheric muons pass through rocks surrounding detector, fast neutrons are produced and the estimated fast neutron background rate is $3.14 \pm 0.09(0.68 \pm 0.04)$ events/day for the ND (FD) [10]. Furthermore, when an energetic muon interacts with ${ }^{12} \mathrm{C}$ in liquid scintillator, unstable isotopes such as ${ }^{9} \mathrm{Li}$ and ${ }^{8} \mathrm{He}$ emitting $(\beta, \mathrm{n})$ followers are produced and mimic IBD signals. Currently, the ${ }^{9} \mathrm{Li} /{ }^{8} \mathrm{He}$ background uncertainty is the largest contribution to the uncorrelated systematic error in the current results and its value is $13.73 \pm 2.13$ (3.61 \pm $0.60)$ events/day for the ND (FD) [10]. By combining these three backgrounds, the total background rate is estimated as $20.48 \pm 2.13(4.89 \pm 0.60)$ events/day for the ND (FD). After subtracting backgrounds, currently daily observed IBD rates are $737.69 \pm 2.57(70.13 \pm 0.74)$ events/day in the ND (FD), respectively [10]. Therefore the level of signal/noise $(\mathrm{S} / \mathrm{N})$ ratio is $\sim 37$ (15) for the ND (FD). The ratio of observed to expected numbers (without oscillation) of antineutrinos in the FD is $0.929 \pm 0.006$ (stat.) \pm 0.009 (syst.). A clear $\sim 7 \%$ of 
disappearance of the reactor antineutrinos in the FD is seen [10]. Further reductions of these backgrounds will continue at RENO by requiring a tighter muon veto cut. Furthermore, a spectral shape analysis will maximize the use of energydependent information in the data.

\section{Physics Reach and Sensitivity of RENO-50}

A relatively large value of $\theta_{13}$ allows us to explore mass hierarchy $(\mathrm{MH})$ and neutrino $\mathrm{CP}$ violation effects $\left(\delta_{\mathrm{CP}}\right)$ from the reactor, accelerator, atmospheric, and very long baseline neutrino experiments. The reactor experiment can determine the neutrino mass hierarchy. Identifying the neutrino mass hierarchy is possible by using a precision measurement of the electron antineutrino survival probability from a nuclear reactor. The survival probability $P\left(\bar{\nu}_{e} \rightarrow \bar{\nu}_{e}\right)$ of the $\bar{\nu}_{e}$ disappearance probability can be written as follows:

$$
\begin{aligned}
1- & P\left(\bar{\nu}_{e} \longrightarrow \bar{\nu}_{e}\right) \\
= & 4 \sum_{j>k}\left|U_{e j}\right|^{2}\left|U_{e k}\right|^{2} \sin \left(\frac{\Delta m_{j k}^{2} L}{4 E}\right) \\
= & \sin ^{2}\left(2 \theta_{13}\right) \sin ^{2}\left(\frac{\Delta m_{31}^{2} L}{4 E}\right) \\
& +\cos ^{4} \theta_{13} \sin ^{2}\left(2 \theta_{12}\right) \sin ^{2}\left(\frac{\Delta m_{21}^{2} L}{4 E}\right) \\
& +\sin ^{2} \theta_{12} \sin ^{2}\left(2 \theta_{13}\right) \cos \left(\frac{\Delta m_{31}^{2} L}{2 E}\right) \sin ^{2}\left(\frac{\Delta m_{21}^{2} L}{4 E}\right) \\
& \pm \frac{1}{2} \sin ^{2} \theta_{12} \sin ^{2}\left(2 \theta_{13}\right) \sin \left(\frac{\Delta m_{31}^{2} L}{2 E}\right) \sin ^{2}\left(\frac{\Delta m_{21}^{2} L}{2 E}\right) .
\end{aligned}
$$

Here, \pm is the mass hierarchy difference. The oscillations are governed by two quadratic mass splittings: $\Delta m_{21}^{2}$ and $\Delta m_{31}^{2}$. Figure 2 shows the $\bar{\nu}_{e}$ disappearance probability as a function of $L$ with the current best values of $\Delta m^{2}$ and $\sin ^{2} 2 \theta_{12}$ and $\sin ^{2} 2 \theta_{13}$ at the upper bound. At smaller $L$, only the $\theta_{13}$ contribution appears. As the distance increases, the $\theta_{12}$ contribution will appear. A large $\theta_{12}$ neutrino oscillation effect appears at $\sim 50 \mathrm{~km}$. In the KamLAND experiment, a $40 \%$ disappearance of $\bar{\nu}_{e}$ was observed at the baseline of $180 \mathrm{~km}$ [17]. Figure 3 shows the reactor neutrino spectrum at $\sim 50 \mathrm{~km}$ for normal hierarchy $(\mathrm{NH})$ and inverted hierarchy (IH). From the large deficit of $\sin ^{2} 2 \theta_{12}, \theta_{12}$ can be precisely measured.

In order to distinguish between normal and inverted mass hierarchy, an extremely good energy resolution of more than $3 \%$ is required, as shown in Figure 4. Energy resolution can be expressed as follows:

$$
\frac{\sigma_{E}}{E}=\frac{a}{\sqrt{E}}+b .
$$

Term $a$ depends on energy resolution. For better energy resolution, we need to increase the number of photoelectrons,

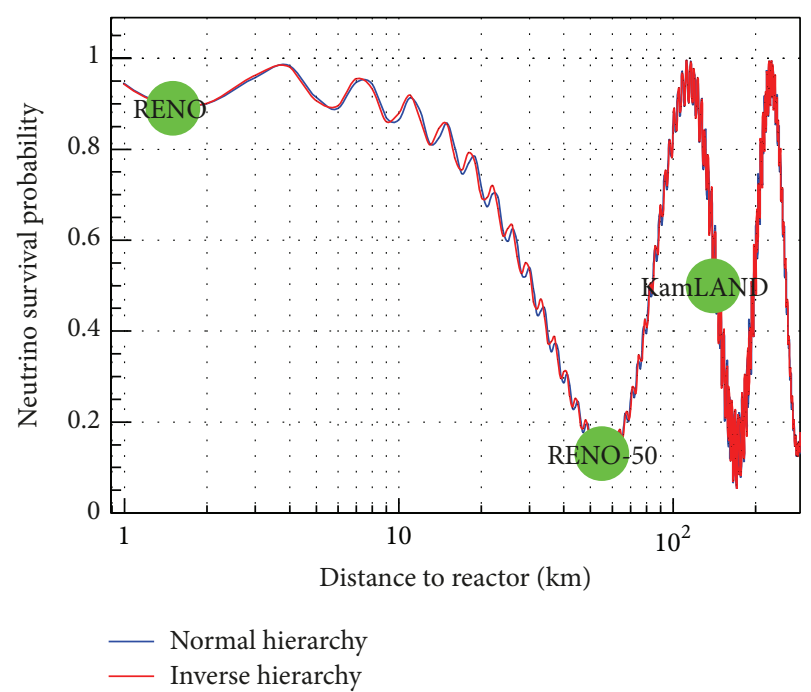

FIGURE 2: Survival probability of $\bar{\nu}_{e}$ as a function of distance $(L)$. Normal hierarchy (NH) and inverted hierarchy (IH) drawn. RENO50 is located at $\sim 50 \mathrm{~km}$ from the reactor center. The KamLAND experiment is $\sim 180 \mathrm{~km}$ away from the reactors.

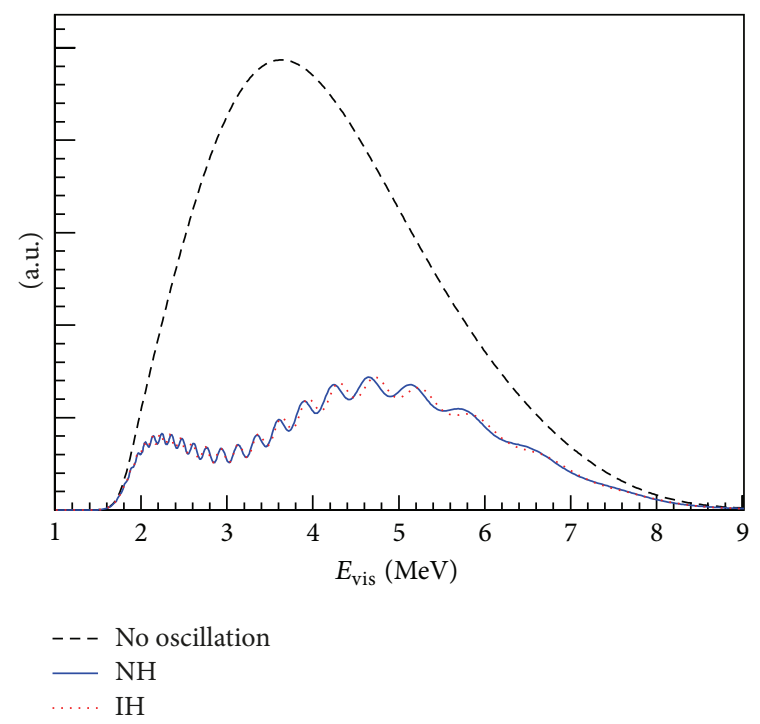

FIGURE 3: Reactor neutrino spectrum at $50 \mathrm{~km}$ for normal hierarchy $(\mathrm{NH})$ and inverted hierarchy $(\mathrm{IH})$. Due to oscillation, deficit of neutrinos will be observed.

$N_{\text {pe }}$. Term $b$ is an energy independent term caused by random processes and is related to PMT noise, thermal noise and electronic noise, and so forth. Currently, the KamLAND energy resolution is at a $\sim 6 \%$ level. In order to achieve 3\% energy resolution, RENO-50 will use high transparency liquid scintillator (LS) which will be used at RENO-50 linear alkyl benzene (LAB, $\mathrm{C}_{n} \mathrm{H}_{2 n+1}-\mathrm{C}_{6} \mathrm{H}_{5}$ ), where $n=10 \sim 13$ is a base candidate solvent for LS [18-21]. Through the careful purification and production process of the base solvent, the attenuation length will be increased from $15 \mathrm{~m}$ to $25 \mathrm{~m}$. Most dirty material used in the LS is PPO $\left(\mathrm{C}_{15} \mathrm{H}_{11} \mathrm{NO}, 2,5\right.$-diphenyloxazole $)$, which is a fluor used to 


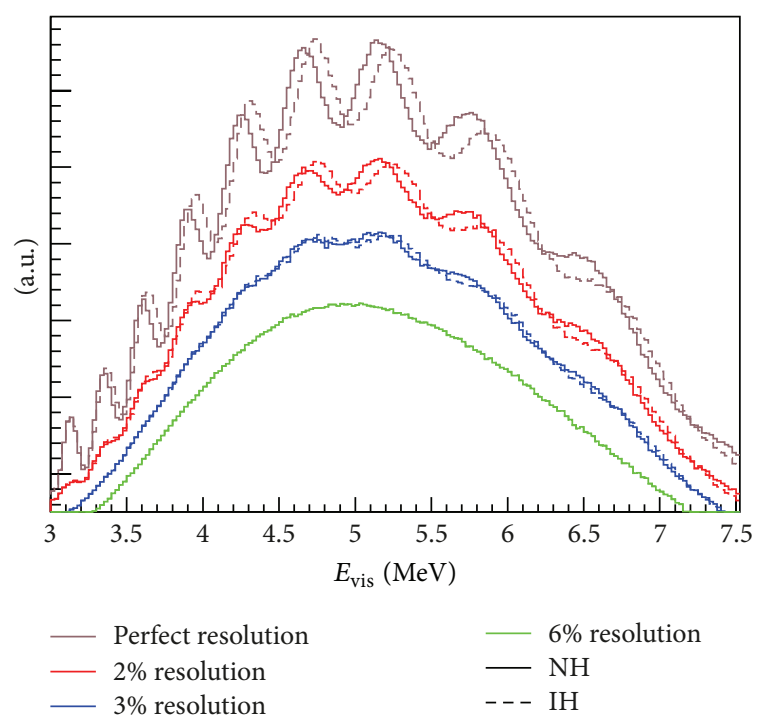

FIGURE 4: Survival probability with different energy resolutions. Solid line refers to $\mathrm{NH}$ and dashed line refers to IH. Energy resolution affects the sensitivity significantly.

produce light emission. By using better quality PPO with no impurities, LS quality will be enhanced. Using 15,000 20-inch PMTs will provide large photocathode coverage from 34\% to 67\%; Hamamatsu 20-inch PMTs will be used, which have an enhanced quantum efficiency (QE) from $20 \%$ to $35 \%$. By adding more PPO $5 \mathrm{~g} / \mathrm{L}$ from $1.5 \mathrm{~g} / \mathrm{L}, 1.5$ times more light yield (LY) in the liquid scintillator can be obtained.

\section{Experimental Site and Detector for RENO-50}

Korea has 4 nuclear reactor power plant sites (Ulchin, Wolsung, Kori, and Yonggwang). RENO-50 is dedicated to the Yonggwang nuclear power plant. The contribution from other nuclear power plants can be negligible. In RENO-50, RENO will be used as a near detector, so that precise reactor neutrino fluxes can be measured. In the KamLAND case, this site is surrounded by 53 Japanese commercial nuclear power plants. Therefore, all of the nuclear reactors are served as a source. A careful survey of the candidate site for RENO-50 has been performed. Several conditions for the selection of the site are required. This should provide a sufficient overburden to reduce cosmic backgrounds. Furthermore, interference among reactor sites and reactor cores significantly affects the sensitivity. The direction of RENO-50 is decided to maximize this sensitivity. A RENO-50 candidate site is shown in Figure 5. An optimal candidate site is at the 450 meter high Mt. Geumseong located in the city of Naju. This corresponds to a $\sim 900$ m.w.e. overburden and it is located $47 \mathrm{~km}$ away from the Yonggwang nuclear power plant.

The RENO-50 detector will use 18,000 tons of ultralowradioactive liquid scintillator (LS) as shown in Figure 6. The diameter of the RENO-50 detector is $30 \mathrm{~m}$ and the height is $30 \mathrm{~m}$ and is 18 times larger than the KamLAND detector. It

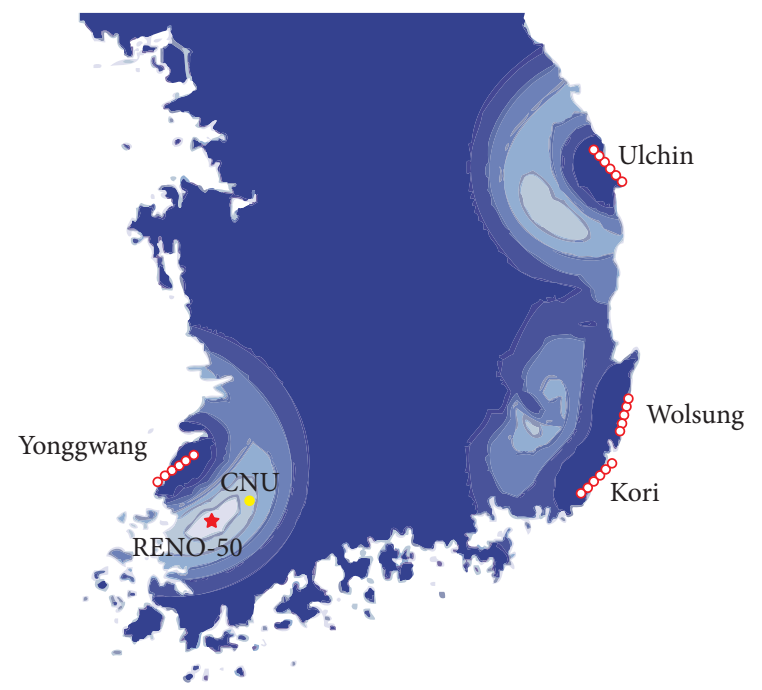

Figure 5: RENO-50 candidate site. The asterisk $(*)$ is a candidate site for RENO-50. The direction to RENO-50 strongly affects the sensitivity. Distribution of four reactors (Ulchin, Wolsung, Kori, and Yonggwang) is considered.

consists of three layers: from the inner to the outer structure, it is target, mineral oil (MO) layer, and the water veto layer. A total of 15,000 20-inch high efficiency PMTs will be installed and this will provide $67 \%$ surface coverage. Based on these detector configurations, the number of neutrino events for a year as a function of the baseline is shown in Figure 7. Observed reactor neutrino rate is estimated as $\sim 15$ events/day. Table 1 shows a comparison between RENO-50 and KamLAND.

\section{Physics with RENO-50}

5.1. Mass Hierarchy. In principle, the mass hierarchy (MH) from precision measurements of $\left|\Delta m_{31}^{2}\right|$ and $\left|\Delta m_{32}^{2}\right|$ can be determined using $\Delta m_{31}^{2}=\Delta m_{32}^{2}+\Delta m_{21}^{2}$. For $\mathrm{NH},\left|\Delta m_{31}^{2}\right|=$ $\left|\Delta m_{32}^{2}\right|+\left|\Delta m_{21}^{2}\right|$. For IH, $\left|\Delta m_{31}^{2}\right|=\left|\Delta m_{32}^{2}\right|-\left|\Delta m_{21}^{2}\right|$. Advantage of reactor neutrino experiments is to determine $\mathrm{MH}$ independently from the $\mathrm{CP}$ phase and matter effects. In addition, a relatively smaller size detector can be employed, unlike the next generation megaton detectors. However, in the RENO-50 case, the determination of $\mathrm{MH}$ is challenging, as it requires an extremely high energy resolution of more than $3 \%$. By using an 18 kton detector, RENO-50 will acquire $\sim 3 \sigma$ significance with 3 years data-taking.

5.2. Precise Measurement of Mixing Parameter $\theta_{12}$ and $\Delta m_{21}^{2}$. In RENO-50, the near and far detectors of RENO could be used as near detectors and thus would reduce the relevant systematic uncertainties significantly. For baselines longer than $50 \mathrm{~km}$, the reactor antineutrino oscillations due to $\Delta m_{31}^{2}$ average out and the survival probability becomes

$$
P=\cos ^{4} \theta_{13}\left[1-\sin ^{2}\left(2 \theta_{12}\right) \sin ^{2}\left(\frac{\Delta m_{21}^{2} L}{4 E}\right)\right] .
$$


TABLE 1: Comparison between RENO-50 and KamLAND.

\begin{tabular}{lccccc}
\hline Experiment & Oscillation reduction & Reactor flux & Detector size & Systematic error (flux) & Error on $\sin ^{2} 2 \theta_{12}$ \\
\hline KamLAND & $40 \%$ & 53 & 1 kton & $3 \%$ & $5.4 \%$ \\
RENO-50 & $77 \%$ & $6 \times 14.7$ & 18 kton & $\sim 0.3 \%$ & $0.4 \%$ \\
\hline
\end{tabular}

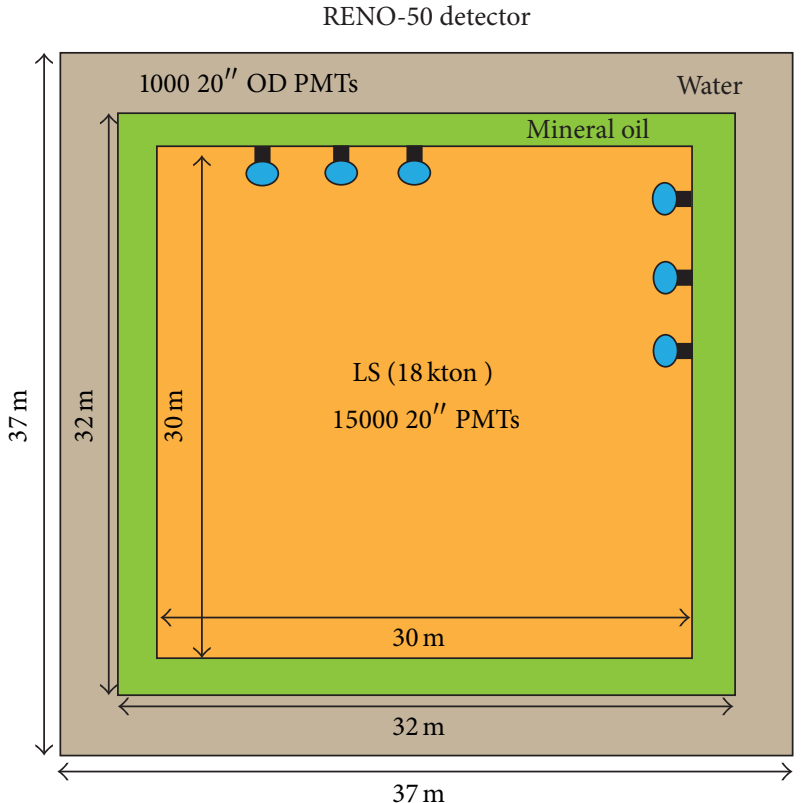

Figure 6: Conceptual design of RENO-50 detector. KamLAND detector is $\sim 1$ kton LS and RENO-50 18 kton LS. 15000 20-inch PMTs will be used. RENO-50 is 18 times larger than the KamLAND detector.

The oscillations due to $\theta_{12}$ and $\Delta m_{21}^{2}$ were observed in the KamLAND experiment $[3,4]$. Because the antineutrino survival probability becomes minimal for $\sin ^{2}\left(\Delta m_{21}^{2} L / 4 E\right) \approx$ 1 , the optimal baseline for measuring $\theta_{12}$ is about $50 \sim 70 \mathrm{~km}$. Namely, $P \approx 1-\sin ^{2} 2 \theta_{12}$ is very sensitive to the value of $\theta_{12}$. The RENO-50 detector is expected to improve the error of the $\theta_{12}$ value. The current value of $\left(\delta \sin ^{2} \theta_{12}\right) /\left(\sin ^{2} \theta_{12}\right)$ is at a $\sim 5.4 \%$ level $[3,4]$. RENO-50 will improve this value to $\sim 1.0 \%(1 \sigma)$ in 1 year. Furthermore, $\left(\delta \Delta m_{12}^{2}\right) /\left(\Delta m_{12}^{2}\right)$ will be improved from the current value of $2.6 \%$ to $\sim 1.0 \%(1 \sigma)$ in 2 years. Figure 8 shows the $\chi^{2}$ distribution as a function of $\sin ^{2} 2 \theta_{12}$.

The sensitivity of the experiment to $\sin ^{2} 2 \theta_{12}$ is calculated using the pull approach through pseudoexperiment [16]. The $\chi^{2}$ function is written as follows:

$$
\begin{aligned}
\chi^{2}= & \sum_{i} \frac{\left[N_{\mathrm{obs}}^{i}-\left(1+a+\xi^{i}+f_{r}\right) N_{\exp }^{i}-\left(1+b_{d}\right) B^{i}\right]^{2}}{N_{\exp }^{i}+B^{i}+\left(\sigma_{b}^{i} B^{i}\right)^{2}} \\
& +\frac{a^{2}}{\sigma_{a}^{2}}+\sum_{i}\left(\frac{\xi^{i}}{\sigma_{\xi}}\right)^{2}+\frac{f_{r}^{2}}{\sigma_{f}^{2}}+\frac{b_{d}^{2}}{\sigma_{b}^{2}} .
\end{aligned}
$$

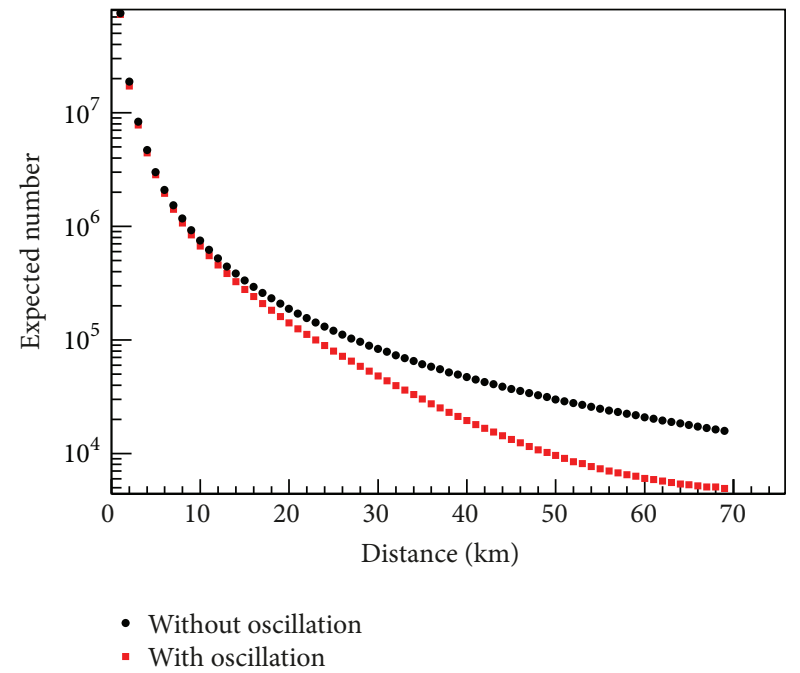

FIGURE 7: Number of neutrino event for a year with oscillation (rectangular) or without oscillation (circle) as a function of baseline from Yonggwang nuclear site.

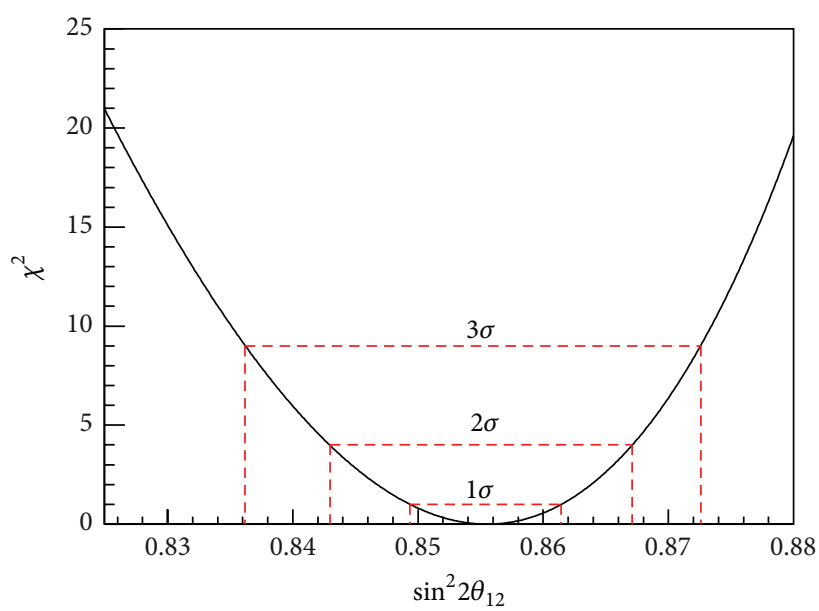

FIgURE 8: $\chi^{2}$ distribution as a function of $\sin ^{2} 2 \theta_{12}$.

$\chi^{2}$ consists of pull terms with observed neutrino events $\left(N_{\text {obs }}^{i}\right)$, expected events $\left(N_{\text {exp }}^{i}\right)$, background $\left(B^{i}\right)$, and systematic uncertainties for neutrino energy bin $i$. Here, $a$ is global normalization and $2.5 \%\left(\sigma_{a}\right)$ uncertainty is used. $\xi^{i}, f_{r}$, and $b_{d}$ are pull parameters. For calculation, detection uncertainty $1.5 \%\left(\sigma_{\xi}\right)$, reactor uncertainty $3 \%\left(\sigma_{f}\right)$, and background uncertainty $5 \%\left(\sigma_{b}\right)$ are used.

5.3. Neutrino Burst from a Supernova. The RENO-50 detector filled with highly purified LS will be sensitive to a burst of neutrinos of all flavors from a Galactic supernova in the 
energy range of a few to tens of $\mathrm{MeV}$. The time scale of the burst is tens of seconds. We assume that the energy of $3.0 \times 10^{53} \mathrm{erg}$ is released during the burst. The background in the RENO-50 detector in a 10 -second period is low enough for an observation of the neutrino signals from the supernova burst. The RENO-50 detector contains $1.35 \times 10^{33}$ free protons, $0.81 \times 10^{33}$ carbons, and $6.21 \times 10^{33}$ electrons. The RENO-50 detector would observe $\sim 4000$ events from a supernova at $8 \mathrm{kpc}[22,23]$. RENO-50 will serve as a longterm astronomical neutrino telescope over 10 years after RENO has recorded data for the $\theta_{13}$ measurement.

5.4. Solar Neutrinos. With an ultralow activity liquid scintillator such as the Borexino level, RENO-50 will search for the matter effect on neutrino oscillation [24]. Therefore, the center of the sun can be probed. Furthermore, the standard solar model would be examined and tested.

5.5. Geoneutrinos. Geoneutrinos are electron antineutrinos produced by $\beta$-decays of ${ }^{238} \mathrm{U},{ }^{232} \mathrm{Th}$, and ${ }^{40} \mathrm{~K}$ decay in the earth's crust and mantle. They provide the surface information on the content of radioactive elements for the entire planet. Their detection can provide information on the sources of the terrestrial heat flow on the present composition and on the origins of the earth. Therefore, the heat generation mechanism inside the earth can be investigated. KamLAND first measured geoneutrinos in 2005 and its value was $40.0 \pm$ 10.5 (stat.) \pm 11.5 (sys.) terrestrial neutrino units (TNU) [25]. In addition, the Borexino detectors are currently collecting geoneutrino data and have reported that the signal rate is $64 \pm 25$ (stat.) \pm 2 (sys.) TNU, after correcting for detection efficiency and the background subtraction [26]. Furthermore, several proposed experiments (e.g., SNO+, Lena, Hanohano, and Earth) will measure geoneutrinos as their primary goals [27-29]. For geoneutrino detection, all experiments use the inverse beta decay (IBD, $\bar{v}_{e}+\mathrm{p} \rightarrow \mathrm{e}^{+}+\mathrm{n}$ ) process. The threshold energy of IBD is $1.8 \mathrm{MeV}$. The measured shape of neutrino spectrum will be essential for determining the observation of geoneutrinos and their radioactive progenitors. The RENO50 detector is large enough for the sensitive geoneutrino measurement and is able to observethem.

5.6. Reactor Neutrino Physics. Currently, RENO observes $\sim 70$ reactor neutrino events per day at the near detector and $\sim 700$ events at the far detector. The RENO and RENO-50 detectors will detect an order of 1 million neutrino events per year. They will measure the flux and energy distribution of the reactor neutrinos with a greater accuracy than previously. This information would then lead to a meaningful comparison of thermal power and reactor fuel loading between measurements and calculations. Such comparison will allow us to measure real-time and direct reactor thermal power with the RENO and RENO-50 detectors. In addition, a precise determination of the reactor neutrino spectrum might be useful for reducing the flux uncertainty [11-13]. Therefore, the reactor neutrinos could be used as an application for the direct monitoring of nuclear fuels and fuel evolution without the need to stop running the nuclear plant.
5.7. Other Physics Topics. Neutrino beams produced from J-PARC (Japan Proton Accelerator Research Complex) in Japan are airborne and appear on Korean peninsula, which is $\sim 1000 \mathrm{~km}$ away from J-PARC. J-PARC beams with an off-axis angle $\left(\sim 3^{\circ}\right)$ can reach the RENO-50 detector at the level of $\sim 400$ per year. Furthermore, RENO-50 will test nonstandard physics such as sterile neutrino physics. The discovery of sterile neutrinos would have a revolutionary impact on neutrino physics [30-34]. While recent neutrino oscillation results are understood in the framework of 3 active neutrino mixings, they do not completely exclude the admixture of sterile neutrinos $[35,36]$. The liquid scintillator neutrino detector (LSND) collaboration implied that gave sterile neutrinos comes from the unconfirmed observation on $v_{\mu} \rightarrow v_{s}$ [37-39]. Mixing with sterile neutrinos based on the LSND signal predicts that the disappearance of the reactor neutrinos with $\Delta m^{2} \sim \mathrm{eV}^{2}$ is very close to the current upper bound from the Bugey experiment [40]. Meanwhile, a scalar field of acceleron associated with the dark energy of the universe implies mass varying neutrinos. Possible couplings of acceleron to matter fields could introduce a very different feature of neutrino oscillation parameters. The mass varying neutrinos could also produce a possible effect in RENO-50. Combined data from the reactor and accelerator neutrino experiments with different path lengths in air and matter will give meaningful information on the mass-varying neutrinos.

\section{Summary of RENO-50 and Outlook}

A surprisingly large value of $\theta_{13}$ will strongly promote the next round of neutrino experiments to find the $\mathrm{CP}$ phase and determine the mass hierarchy. The main goals of RENO-50 are to measure the most accurate (1\%) value of $\theta_{12}$ and to attempt to determine the neutrino mass hierarchy. RENO50 is expected to detect neutrinos from nuclear reactors, the Sun, Supernova, the Earth, any possible stellar objects, and J-PARC neutrino beam. It could act as a neutrino telescope.

RENO-50 is a long-term operational and multipurpose detector. A candidate site has been found at the 450 meter high Mt. Geumseong, $47 \mathrm{~km}$ from the Hanbit nuclear power plant. RENO-50 requires an inclined tunnel to obtain a deeper location. It will use 18,000 tons of ultralowradioactivity unloaded liquid scintillator and 15,000 20inch high quantum efficiency PMTs with a $67 \%$ surface coverage. The current RENO detectors can serve as near detectors. The sensitivities are studied to determine the $\mathrm{MH}$. Determining $\mathrm{MH}$ is very challenging, but not impossible with very good energy resolution of more than a $3 \%$ level. In addition, neutrino oscillation parameters, $\theta_{12}$ and $\Delta m_{12}^{2}$, will be precisely measured at less than a $0.5 \%$ level, which can constrain new physics. In summary, the RENO-50 reactor experiment with the medium baseline of $\sim 50 \mathrm{~km}$ is expected to perform high-precision measurements of $\theta_{12}, \Delta m_{21}^{2}$, and $\Delta m_{31}^{2}$ and to determine the mass hierarchy. It will provide nuclear fuel monitoring with reactor neutrinos. Reactor experiments have played and will play an important role in both new discoveries and precision measurements in the neutrino sector. Therefore, a complete understanding of 
neutrino oscillation will provide an opportunity to explore new physics.

\section{Conflict of Interests}

The authors declare that there is no conflict of interests regarding the publication of this paper.

\section{Acknowledgments}

This work was supported by the Korea Neutrino Research Center, which was established by the National Research Foundation of Korea (NRF) Grant funded by the Korea Government (MSIP) (no. 2009-0083526) and the Basic Science Research Program through the National Research Foundation of Korea (NRF) funded by the Ministry of Education (2010-0007850, 2012-0001177, and 2012M2B2A6030210).

\section{References}

[1] B. Pontecorvo, "Neutrino experiments and the problem of conservation of leptonic charge," Journal of Experimental and Theoretical Physics, vol. 53, pp. 1717-1725, 1967.

[2] Z. Maki, M. Nakagawa, and S. Sakata, "Remarks on the unified model of elementary particles," Progress of Theoretical Physics, vol. 28 , no. 5, pp. 870-880, 1962.

[3] K. Eguchi, S. Enomoto, K. Furuno et al., "First results from KamLAND: evidence for reactor antineutrino disappearance," Physical Review Letters, vol. 90, no. 2, Article ID 021802, 6 pages, 2003.

[4] T. Araki, K. Eguchi, S. Enomoto et al., "Measurement of neutrino oscillation with KamLAND: evidence of spectral distortion," Physical Review Letters, vol. 94, no. 8, Article ID 081801, 5 pages, 2005.

[5] E. Aliu, S. Andringa, S. Aoki et al., "Evidence for muon neutrino oscillation in an accelerator-based experiment," Physical Review Letters, vol. 94, no. 8, Article ID 081802, 5 pages, 2005.

[6] M. H. Ahn, S. Aoki, H. Bhang et al., "Indications of neutrino oscillation in a $250 \mathrm{~km}$ long-baseline experiment," Physical Review Letters, vol. 90, no. 4, Article ID 041801, 5 pages, 2003.

[7] M. Apollonio, A. Baldini, C. Bemporad et al., "Search for neutrino oscillations on a long base-line at the chooz nuclear power station," The European Physical Journal C, vol. 27, no. 3, pp. 331-374, 2003.

[8] Y. Abe, C. Aberle, J. C. dos Anjos et al., "Reactor electron antineutrino disappearance in the Double Chooz experiment," Physical Review D, vol. 86, no. 5, Article ID 052008, 21 pages, 2012.

[9] F. P. An, J. Z. Bai, A. B. Balantekin et al., "Observation of electron-antineutrino disappearance at daya bay," Physical Review Letters, vol. 108, no. 17, Article ID 171803, 7 pages, 2012.

[10] J. K. Ahn, S. Chebotaryov, J. H. Choi et al., "Observation of reactor electron antineutrinos disappearance in the RENO experiment," Physical Review Letters, vol. 108, no. 19, Article ID 191802, 6 pages, 2012.

[11] T. A. Muller, D. Lhuillier et al., "Improved predictions of reactor antineutrino spectra," http://arxiv.org/abs/arXiv:1101.2663.

[12] A. A. Hahn, K. Schreckenbach, W. Gelletly, F. von Feilitzsch, G. Colvin, and B. Krusche, "Antineutrino spectra from ${ }^{241} \mathrm{Pu}$ and
${ }^{239} \mathrm{Pu}$ thermal neutron fission products," Physics Letters B, vol. 218, no. 3, pp. 365-368, 1989.

[13] G. Mention, M. Fechner, T. Lasserre et al., "Reactor antineutrino anomaly," Physical Review D, vol. 83, no. 7, Article ID 073006, 20 pages, 2011.

[14] K. Abe, T. Akin, N. Abgrall et al., "Recent results from the T2K experiment," Nuclear Physics B, vol. 246-247, pp. 23-28, 2014.

[15] http://www.mpi-hd.mpg.de/personalhomes/globes.

[16] J. Ahn, S. R. Baek, S. Choi et al., "RENO: an experiment for neutrino oscillation parameter $\theta_{13}$ using reactor neutrinos at Yonggwang," http://arxiv.org/abs/1003.1391.

[17] K. Eguchi, S. Enomoto, K. Furuno et al., "A High sensitivity search for anti-nu(e)'s from the sun and other sources at KamLAND," Physical Review Letters, vol. 92, Article ID 071301, 2004.

[18] J. S. Park, S. B. Kim, J. Lee, B. C. Kim, S. H. Kim, and K. K. Joo, "Feasibility study of a liquid scintillator using domestically produced Linear Alkyl Benzene (LAB)," New Physics, vol. 58, no. 1, pp. 62-67, 2009.

[19] I. S. Yeo and K. K. Joo, "Study on the optical properties of a linear alkyl benzene-based liquid scintillator in the RENO," New Physics, vol. 61, no. 8, pp. 739-743, 2011.

[20] J. S. Park, J. Lee, I.S. Yeo et al., "Production and optical properties of Gd-loaded liquid scintillator for the RENO neutrino detector," Nuclear Instruments and Methods in Physics Research A, vol. 707, pp. 45-53, 2013.

[21] B. K. Kim, S. H. So, S. H. Song, and K. K. Joo, "Investigation of the physical and optical properties of various base solvents for the liquid scintillator in a neutrino detector," Journal of Korean Physical Society, vol. 62, no. 1, pp. 26-33, 2013.

[22] K. Scholberg, "Supernova neutrino detection," http://arxiv.org/ abs/astro-ph/0701081.

[23] L. Cadonati, F. P. Calaprice, and M. C. Chen, "Supernova neutrino detection in Borexino," Astroparticle Physics, vol. 16, no. 4, pp. 361-372, 2001.

[24] G. Alimonti, C. Arpesella, M. Balata et al., "Light propagation in a large volume liquid scintillator," Nuclear Instruments and Methods in Physics Research A, vol. 440, no. 2, pp. 360-371, 2000.

[25] T. Araki, S. Enomoto, K. Furuno et al., "Experimental investigation of geologically produced antineutrinos with KamLAND," Nature, vol. 436, pp. 499-503, 2005.

[26] G. Fiorentini, G. L. Fogli, E. Lisi, F. Mantovani, and A. M. Rotunno, "Mantle geoneutrinos in KamLAND and borexino," Physical Review D, vol. 86, no. 3, p. 033004, 2012.

[27] J. Hahn, R. L. Boger, J.K. Rowleyand et al., "The sudbury neutrino observatory," Nuclear Instruments and Methods in Physics Research A, vol. 449, no. 1-2, pp. 172-207, 2000.

[28] G. Fiorentini, M. Lissia, and F. Mantovani, "Geo-neutrinos and earth's interior," Physics Reports, vol. 453, no. 5-6, pp. 117-172, 2007.

[29] A. Mazumdar and M. M. Sheikh-Jabbari, "Noncommutativity in space and primordial magnetic field," Physical Review Letters, vol. 87, no. 1, Article ID 011301, 4 pages, 2001.

[30] D. O. Caldwell and R. N. Mohapatra, "Neutrino mass explanations of solar and atmospheric neutrino deficits and hot dark matter," Physical Review D, vol. 48, no. 7, pp. 3259-3263, 1993.

[31] J. T. Peltnoniemi, D. Tommasini, and J. W. F. Valle, "Reconciling dark matter and solar neutrinos," Physics Letters B, vol. 298, pp. 383-390, 1993. 
[32] J. T. Peltnoniemi and J. W. F. Valle, "Reconciling dark matter, solar and atmospheric neutrinos," Nuclear Physics B, vol. 406, no. 1-2, pp. 409-422, 1993.

[33] K. Benakli and A. Y. Smirnov, "Neutrino-modulino mixing," Physical Review Letters, vol. 79, no. 22, pp. 4314-4317, 1997.

[34] B. Kayser, "Neutrino mass: where do we stand, and where are we going?" http://arxiv.org/abs/hep-ph/9810513.

[35] G. Mention, M. Fechner, M. Cribier et al., "The reactor antineutrino anomaly," http://arxiv.org/abs/arXiv:1101.2755.

[36] T. A. Muller, D. Lhuillier, M. Fallot et al., "Improved predictions of reactor antineutrino spectra," http://arxiv.org/abs/arXiv:1101. 2663.

[37] C. Athanassopoulos, L. B. Auerbach, R. L. Burman et al., "Evidence for $\overline{\nu_{e}} \rightarrow \overline{\nu_{e}}$ oscillations from the LSND experiment at the los alamos meson physics facility," Physical Review Letters, vol. 77, no. 15, pp. 3082-3085, 1996.

[38] A. Aguilar, L. B. Auerbach, and R. L. Burman, "Evidence for neutrino oscillations from the observation of $\bar{v}_{e}$ appearance in a $\bar{\nu}_{\mu}$ beam," Physical Review D, vol. 64, no. 11, Article ID 112007, 22 pages, 2001.

[39] C. Athanassopoulos, L. B. Auerbach, and R. L. Burman, "Results on $v_{\mu} \rightarrow v_{e}$ neutrino oscillations from the LSND experiment," Physical Review Letters, vol. 81, p. 1774, 1998.

[40] M. Maltoni, T. Schwetz, M. A. Tórtola, and J. W. F. Valle, "Ruling out four-neutrino oscillation interpretations of the LSND anomaly?" Nuclear Physics B, vol. 643, no. 1-3, pp. 321$328,2002$. 

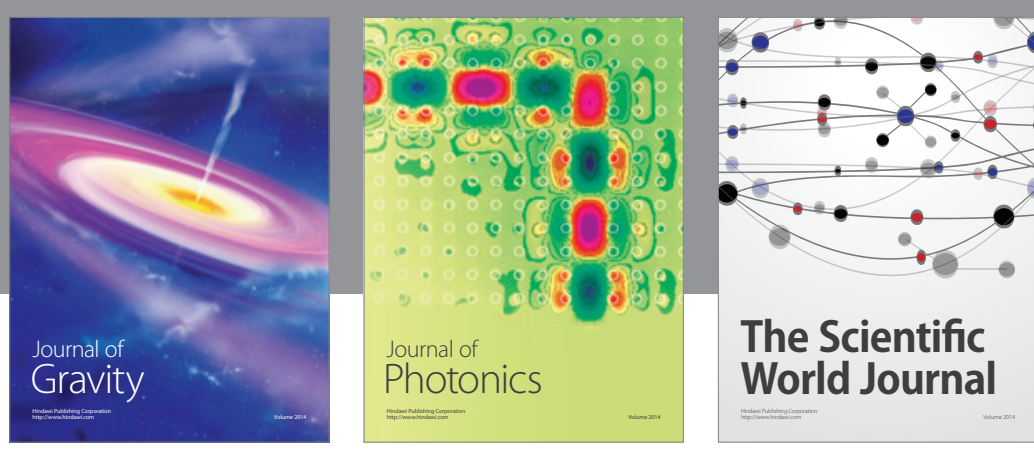

The Scientific World Journal
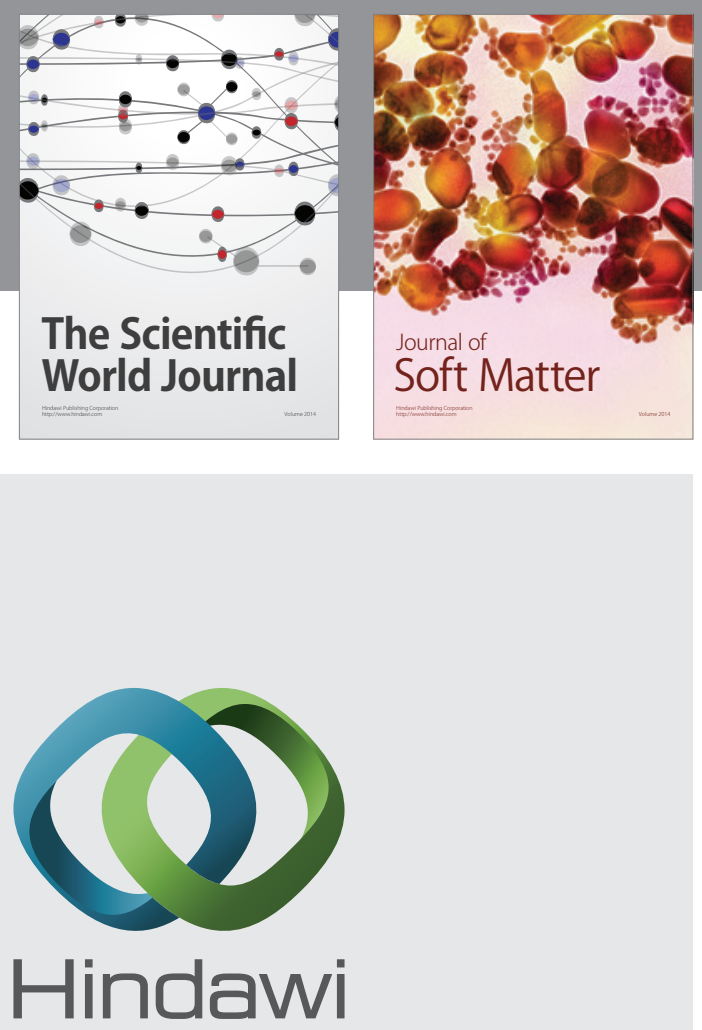

Submit your manuscripts at

http://www.hindawi.com

nternational Journal of

Statistical Mechanics
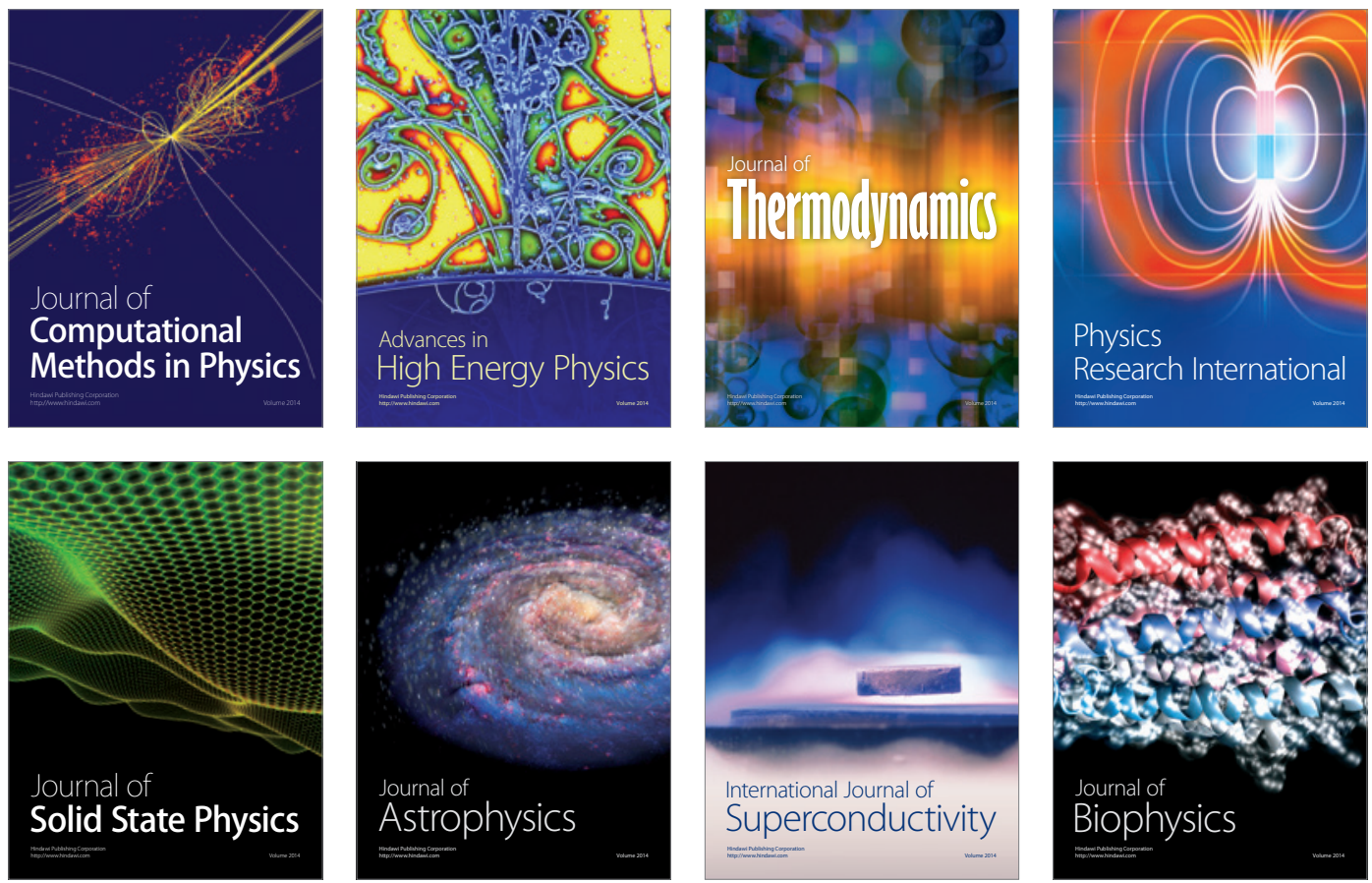
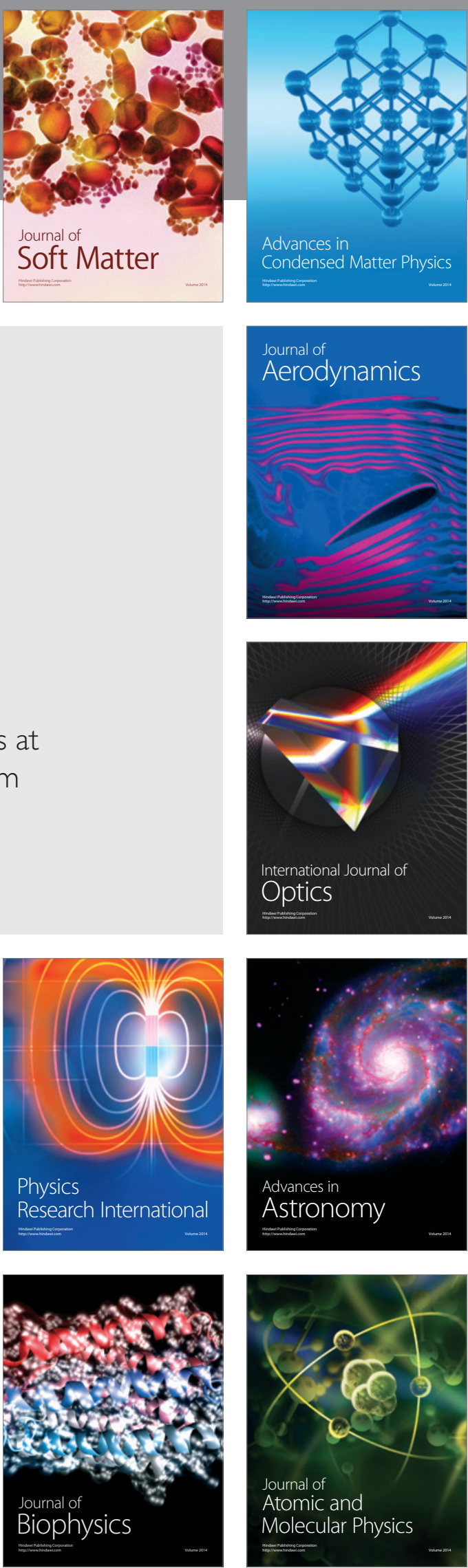